1 Article

\title{
2 A New Paradox in Quantum Mechanics
}

\section{Ghenadie N. Mardari 1,*}

1 OWR, Sparks, Maryland, USA

* Correspondence: gmardari@gmail.com; Tel.: +1-443-465-4350

Abstract: The EPR paradox is known as an interpretive problem, as well as a technical discovery in quantum mechanics. It defined the basic features of two-quantum entanglement, as needed to study the relationships between two non-commuting variables. In contrast, four variables are observed in a typical Bell experiment. This is no longer the same problem. The full complexity of this process can only be captured by the analysis of four-quantum entanglement. Indeed, a new paradox emerges in this context, with straightforward consequences. Either quantum behavior is capable of signaling non-locality, or it is local. Both alternatives appear to contradict existing knowledge. Still, one of them has to be true, and the final answer can be obtained conclusively with a four-quantum Bell experiment.

Keywords: Bell's theorem; EPR paradox; quantum entanglement; non-locality.

\section{Introduction}

In quantum mechanics, Bell-type inequalities are tested by conducting a series of joint measurements over a common source of emitted particles. For example, the CHSH protocol [1] contains a cycle of four paired observations, such as $(\mathbf{A}, \mathbf{B}),(\mathbf{B}, \mathbf{C}),(\mathbf{C}, \mathbf{D})$, and $(\mathbf{D}, \mathbf{A})$. It seems reasonable to assume that all of these measurements study the same input population. Therefore, their outcomes should be described as combinations of four random variables sampling a unique hidden variable " $\lambda$ ". Unfortunately, this interpretation does not work in quantum mechanics, at least in the case of non-commuting variables, because Bell-type inequalities are consistently violated [2-7]. Instead, it is more accurate to suggest that joint measurement $(\mathbf{A}, \mathbf{B})$ samples the hidden variable " $\boldsymbol{\lambda}_{\mathbf{1}}$ ", joint measurement $(\mathbf{B}, \mathbf{C})$ samples the hidden variable " $\boldsymbol{\lambda}_{2}$ ", and so on. In other words, we are forced to assume that every joint measurement is performed over a different population [8], even though the same physical source of quanta is employed and all the precautions are taken to avoid selection bias. How is this possible?

There are two known ways to produce observables in violation of Bell's inequality. Yet, both of them appear problematic for quantum behavior. On the one hand, it is possible to sample different populations because of some unsuspected filtering process. For instance, consider a public survey in which questions $\mathbf{A}$ and $\mathbf{B}$ are answered only by men, while questions $\mathbf{B}$ and $\mathbf{C}$ are answered only by women. Presumably, men and women took the survey with equal frequency, but they chose not to answer all the questions, and only coincident results were counted. Hence, every joint measurement is actually performed over a different group of people. On the other hand, it is also possible to sample the same individuals, only to discover that the answer to each question is context-dependent. For instance, consider a public survey in which asking question $\mathbf{A}$ before $\mathbf{B}$ produces one outcome, while asking question $\mathbf{B}$ before $\mathbf{A}$ produces a different outcome. Hence, the same group of people is measured, but the underlying relevant distribution is different. Strictly speaking, the same questions correspond to different random variables in each context [8]. In quantum theory, the first scenario (with hidden filtration) is often considered unreasonable, because of the amount of efforts expended over several decades to close various "loopholes" [9-17]. However, the second scenario (with non- 
local contexts) cannot be given a straightforward physical interpretation, because it has to be both "non-local" and "non-signaling" [18-21]. (If non-locality is real, why does it not have any directly observable implication?) Here we present a new paradox that brings out the key nuances of these concepts and, more importantly, opens up the possibility of experimental verification. As will be shown below, coincidence experiments with four-quantum entanglement should be able to expose the true source of Bell violations. Therefore, it might be possible to determine, once and for all, if quantum behavior is local or non-local.

\section{The Paradox}

A set of variables cannot have joint distributions, if their correlations violate Bell-type inequalities [22-24]. For an intuitive illustration, consider a large "population" of shirts, measured for three binary properties: fabric (cotton/ non-cotton), color (white/ non-white), and sleeve length (short/ long). Suppose that a measurement procedure yields the following rules of association between the properties:

1. All the cotton shirts are white.

2. All the white shirts are short-sleeved.

3. All the cotton shirts are long-sleeved.

There is an obvious contradiction between these three sets of coincidences. If all the cotton shirts are white and all the white shirts are short-sleeved, then all the cotton shirts should be short-sleeved. The three correlations could not have been registered in the same population. At least one of the three joint measurements must have taken place in a different context. It is precisely this sort of incompatibility that leads to violations of Bell-type inequalities. Moreover, this conclusion would follow even if only some shirts (rather than all of them) were found to have contradictory properties.

Though, how is it possible to get this kind of observations with a common group of objects? If all the variables of interest are measured together, then joint distributions are obtained by default. Therefore, Bell violations can only be detected if various pairwise correlations are tested independently from each other. This means that every quality of interest must be measured repeatedly. Individual properties can only have contradictory values, if they have the opportunity to display them in sequence. Absent such a mechanism, violations are impossible in any environment, with or without non-locality. For example, in a typical Bell experiment (using the CHSH inequality), there are four joint measurements:

$$
\mathrm{S}=\mathrm{E}(\mathbf{A}, \mathbf{B})+\mathrm{E}(\mathbf{B}, \mathbf{C})+\mathrm{E}(\mathbf{C}, \mathbf{D})-\mathrm{E}(\mathbf{D}, \mathbf{A}) \text {. }
$$

Every variable is part of two combinations, therefore it must be measured twice. This nuance is often overlooked, because Bell experiments are usually performed with two-quantum entanglement. In this sort of settings, it is only possible to measure two properties at a time. This limitation forces the observers to repeat every measurement, making it seem as if this extra step is just a technicality. If quantum mechanics predicts a certain type of correlations, shouldn't it be the same in every similar measurement? This is a very important question to ask, because the process of entanglement is not restricted to the two-quantum case. Indeed, four-quantum entanglement has recently become a hot topic of research [25-27], because of its practical advantages in quantum communication protocols. Ergo, it is possible to conduct an experiment in which all the four variables of a Bell test are measured at the same time. Logically, this means that one and the same distribution for each property can be used for every combination, in order to determine the coefficients of correlation. At the individual level, one value of observable $\mathbf{A}$ is used for the coincidence with $\mathbf{B}$, and the same value is used for the coincidence with $\mathbf{D}$. Yet, how can the same value contradict itself? This is patently impossible. Therefore, such a protocol can never produce violations of any Bell-type inequality.

In light of this conclusion, quantum mechanics appears to contain a new paradox. If quantum variables are measured two at a time with four-quantum entanglement (by ignoring the values of two out of four quanta), then we should expect violations of the CHSH inequality, just like in any other Bell experiment. Yet, such violations are impossible if all the four quanta are measured at the same time, deriving the coefficients of correlation from the record of quadruple coincidences. Both of these predictions must follow with accuracy from the formalism of quantum mechanics, if it is 
presumed to be a self-consistent theory. Though, how can they take place at the same time, in the same experimental setting? It looks as if the rules of association between the values of two quantum variables depend on the observer's decision to measure or not to measure two other variables, even if the corresponding quanta are detected at immense distances from each other. This is similar to the EPR paradox for two-quantum entanglement, but this time the ontological implications are directly observable. Instead of guessing that individual properties change between measurements, we can look for actual differences in the record of events, because the observed coefficients of correlation have to change from case to case.

\section{The Solution}

Four-quantum entanglement is a relatively recent development in quantum mechanics, but there are numerous instructive demonstrations already [25-27]. For the purpose of this argument, consider a hypothetical source of quadruple entanglement, emitting four streams of correlated particles in different directions (Fig. 1A). Suppose that individual properties cannot be determined in advance, but they are certain to be identical for all the quanta in a set, if measured in the same way. In the case of dichotomous variables, only two joint measurement outcomes are possible: $(1,1,1,1)$ or $(0,0,0,0)$. This means that any two quanta, selected from a set of four, would behave as a typical entangled pair in the singlet state, with possible outcomes $(1,1)$ and $(0,0)$. Ergo, they should be expected to generate coincidences in violation of the $\mathrm{CHSH}$ inequality, if they are measured as needed for this protocol. Yet, the experimental arrangement allows for the simultaneous measurement of four observables at the same time. As seen in Fig. 1A, quantum 1 can be measured for property $\mathbf{A}$, while quantum 2 for property $\mathbf{B}$, and so on. The same record of detection events can be used to calculate all the necessary coefficients of correlation for a $\mathrm{CHSH}$ protocol. Yet, as shown above, Bell violations can only take place if individual properties are measured twice. If all the data is obtained in one iteration, then all the four variables are jointly distributed by default. Accordingly, the coefficients of correlation for each combination must be able to violate and to not violate the CHSH inequality at the same time. Both types of outcomes are predicted by quantum theory. Yet, this can only happen if observable coefficients of correlation are incompatible with themselves. They should be able to violate Bell-type inequalities when two randomly chosen quanta are ignored. However, they should also be unable to do so when all the four quanta in a set are taken into account.

At first sight, the best way to solve this paradox is by invoking some sort of non-local mechanism. Perhaps, quantum behavior is such that a choice between measuring only two or all four entangled quanta results in different correlations? The problem is that such a hypothesis can only work if it allows exclusively for non-signaling non-locality [33-35], but no such assurance is available in the case of four-quantum entanglement. For instance, it is possible to set up a cosmic experiment, in which two streams of photons are sent to the Moon, while the other two are kept on Earth, in fiber optic loops. Two seconds after launching the quanta, terrestrial observers can decide whether to measure or to discard their photons. If non-locality is real, then the open space channels should be instantly affected. Accordingly, Moon-based observers can determine if the remote channels were recorded or not by performing a Bell test with their two streams of photons. This would make it possible to establish superluminal communications between the Earth and the Moon. Ergo, non-local explanations of four-quantum entanglement cannot be compatible with quantum mechanics. There is an instructive precedent for this problem, known as Poppers' experiment [28, 29]. If non-local collapse is assumed to work at the level of single particles, then - it seems - EPR measurements of momentum should display signaling non-locality. Yet, the experiment did not confirm this expectation [28]. More importantly, formal analysis did not support it either [29]. Quantum predictions are derived from the net effect of superposed wave-function components. It is a mistake to interpret them with models that only consider particle-type behavior. Furthermore, quantum mechanics was built around the correspondence principle [30]. Its predictions for large- $N$ observations cannot contradict well-established macroscopic facts. If Bell violations were due to signaling non-locality, this would be observable at classical levels of radiation as well. 


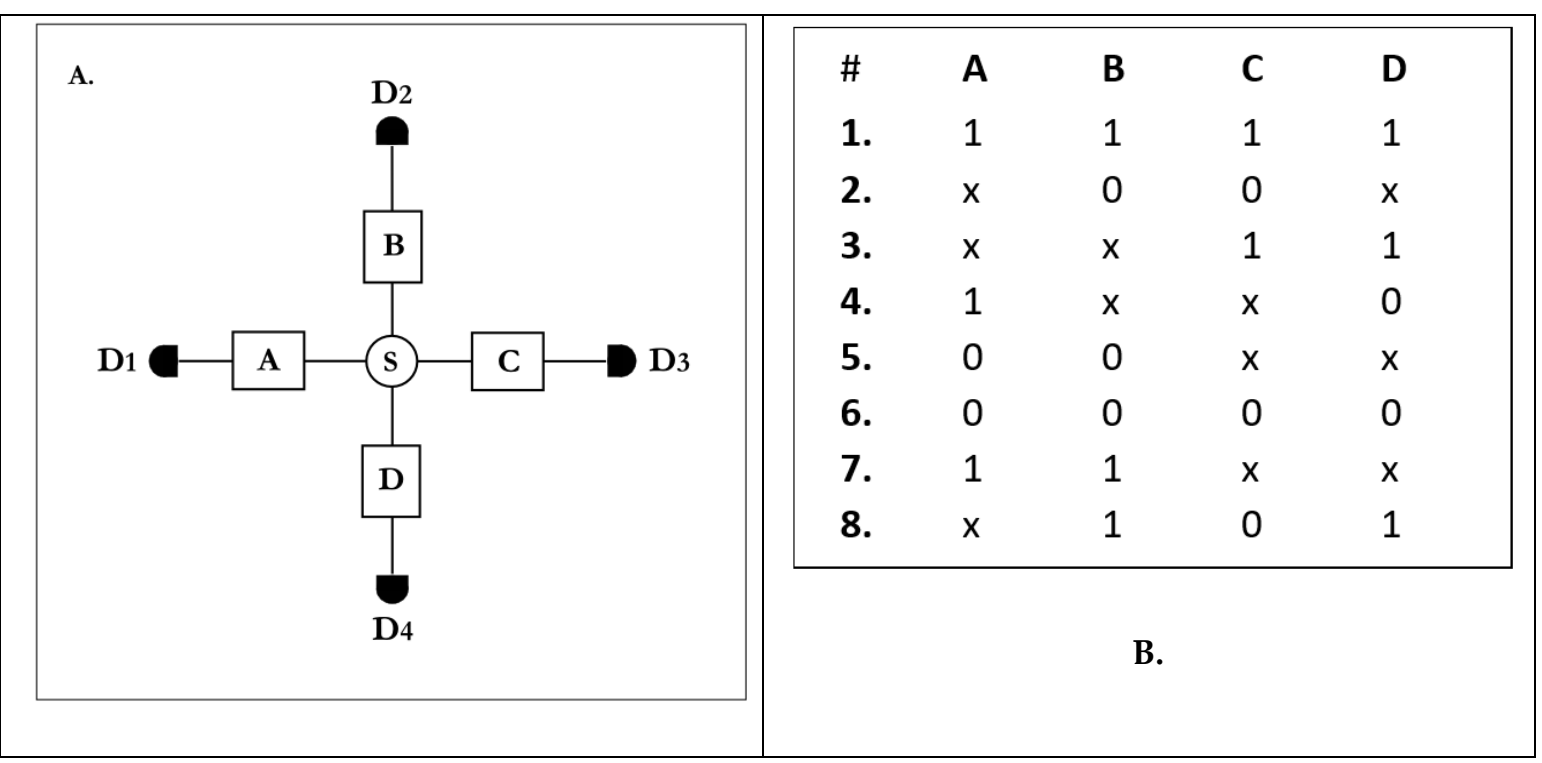

Fig.1. Four-quantum entanglement without paradox.

A) Conceptual diagram. Four entangled quanta pro-pagate in opposite directions from a common (virtual) source-point. The marked boxes (A, B, C, and D) symbolize four different measurement procedures, corresponding to four non-commuting quantum variables. When measured in the same way, all the four quanta have an equal probability of detection. When measured in different ways, these probabilities are individually determined by input parameters.

B) Hypothetical data plot, illustrating possible patterns of detection, when Bell-type inequalities are violated without paradox. Values " 0 " and " 1 " represent measurement outcomes, while " $x$ " corresponds to missing events. Four-fold coincidences correspond to a minority of trials (in this case, lines 1 and 6), while the majority of trials produce two-fold coincidences, in various combinations. This is why different pairs of observables end up sampling different populations.

The puzzle of four-quantum entanglement can have a physically sound solution, if it has a logical solution. So, we have to inquire: what kind of configuration would make the contradictions go away? It has to be true that pair-wise detection violates Bell-type inequalities. It must also be true that quadruple detection does not violate the same inequalities, in identical experimental settings. Is it possible for both of these processes to take place at the same time, if we are free to make any convenient assumption? Firstly, suppose that the number of double coincidences is equal to the number of quadruple coincidences (meaning that all the quanta are detected in ideal experiments). In this case, it is impossible for both outcomes to be true. Either Bell-type inequalities are violated, or not. The paradox stands. Secondly, suppose that the number of quadruple coincidences is larger than the number of double coincidences. This hypothesis must be dismissed as unsound, because quadruple coincidences also include double coincidences. Finally, consider the possibility that double coincidences outnumber quadruple coincidences. In this case, the paradox vanishes. We can envision an experiment in which a minority of quantum sets produce four coincident events, but most of them produce only two. Bell-type inequalities cannot be violated by the subgroup of quanta that generate quadruple detections. However, every pairwise coincidence (above the four-event threshold) is free from this constraint. If every type of pairwise coincidence belongs to a well-defined slice of the input group of sampled entities, then they can have stable coefficients of correlation. Therefore, Bell-type inequalities can be violated, because the underlying populations are different for each combination of measurements.

For clarity, quantum experiments are often designed to reveal information about individual modes of propagation from multi-mode input beams. Four-quantum entanglement would be paradox-free if its variables were also defined as properties of wave-function components, without representing the full spectrum of a wave-function. In particular, one could assume that measurement 
settings determine which components become observable. If so, then changing these settings may alter the subset of detectable quanta. Hence, all the members of an entangled four-quantum set should be detectable if measured in the same way. In contrast, if every quantum is measured in a different way, then some members from each group should be likely to miss their detectors, depending on the input component that they represent. For example, some sets might generate coincidences for $\mathbf{A}$ and $\mathbf{B}$, but not for $\mathbf{C}$ and $\mathbf{D}$; others for $\mathbf{B}$ and $\mathbf{C}$ only, and so on. In process terms, some sets might generate only one detection event or none, others might generate two or three coincident events, and only a minority would generate quadruple detections (Fig. 1B). The formalism of quantum mechanics would be entirely self-consistent, if it could predict all of these rates of coincidence with precision. Yet, this raises another question: how can something like this be true, when every known Bell experiment was explicitly designed to avoid sampling bias?

In conclusion, quantum mechanics is confronted with a tough choice, going forward: either quantum behavior is inherently paradoxical (in which case signaling non-locality should be observable), or it is perfectly self-consistent (in which case local models of quantum behavior should be reconsidered). Four-quantum entanglement does not seem to allow for a compromise between these two alternatives. However, it makes it possible to verify directly the underlying mechanism for Bell violations. Either one of the two outcomes is likely to produce new questions, since they are both in apparent conflict with existing knowledge. Nonetheless, it is inspiring to see that the debates about the nature of quantum reality do not have to be interminable.

Acknowledgments: The final shape of this argument was greatly influenced by discussions with France Čop, Marcus Appleby, Ehtibar Dzhafarov, and Ligia Mardari. I am also grateful to Andrei Khrennikov, Guillaume Adenier, Sören Wengerowsky, Giuliano Scarcelli and Robert Boyd for helpful explanations and references. Finally, credit is also due to the participants of the Växjö Conference series on Quantum Foundations, as well as the SPIE Optics and Photonics series, taking place every summer in Sweden and the USA, respectively.

Conflicts of Interest: The author declares no conflict of interest.

\section{References:}

1. Clauser, J. F.; Horne, M. A.; Shimony, A.; Holt, R. A. Proposed experiment to test local hidden-variable theories. Phys. Rev. Lett. 1969, 23, p. 880.

2. Bell, J. S. Speakable and Unspeakable in Quantum Mechanics; Cambridge, 1987.

3. Afriat, A.; Selleri, F. The Einstein, Podolsky, and Rosen Paradox in Atomic, Nuclear, and Particle Physics; Springer, 1998.

4. Bertlman, R. A.; Zeilinger, A. (eds). Quantum [Un]speakables: From Bell to Quantum Information; Springer, 2002.

5. Wheeler, J. A.; Zurek, W. H. (eds). Quantum Theory and Measurement; Princeton, 1983.

6. Peres, A. Quantum Theory: Concepts and Methods; Kluwer, 1993.

7. Zeilinger, A. Experiment and the foundations of quantum physics. Rev. Mod. Phys. 1999, 71, p. S288.

8. Dzhafarov, E. N.; Kujala,J. V. Context-content systems of random variables: The contextuality-by-default theory. J. Math. Psych. 2016, 74, p. 11.

9. Aspect, A.; Grangier, P.; Roger, G. Experimental tests of realistic local theories via Bell's theorem. Phys. Rev. Lett. 1981, 47, p. 460.

10. Aspect, A.; Grangier, P.; Roger, G. Experimental realization of Einstein-Podolsky-Rosen-Bohm gedankenexperiment: a new violation of Bell's inequalities. Phys. Rev. Lett. 1982, 49, p. 91.

11. Aspect, A.; Dalibard, J.; Roger, G. Experimental test of Bell's inequalities using time-varying analyzers. Phys. Rev. Lett. 1982, 49, p. 1804.

12. Rowe, M. A.; et al. Experimental violation of a Bell's inequality with efficient detection. Nature 2001, 409, p. 791.

13. Christensen, B. G.; et al. Detection-loophole-free test of quantum nonlocality, and applications. Phys. Rev. Lett. 2013, 111, 130406.

14. Giustina, M.; et al. Bell violation using entangled photons without the fair-sampling assumption. Nature 2013, 497, p. 227.

15. Hensen, R.; et al. Loophole-free Bell inequality violation using electron spins separated by 1.3 kilometres. Nature 2015, 526, p. 682. 
16. Giustina, M.; et al. Significant-loophole-free test of Bell's theorem with entangled photons. Phys. Rev. Lett. 2015, 115, 250401.

17. Shalm, L. K.; et al. A strong loophole-free test of local realism. Phys. Rev. Lett. 2015, 115, 250402.

18. Popescu, S.; Rohrlich, D. Quantum nonlocality as an axiom. Found. Phys. 1994, 24, p. 379.

19. Ghirardi, G. C.; Rimini, A.; Weber, T. A. General argument against superluminal transmission through the quantum mechanical measurement process. Lett. Nuovo Cimento. 1980, 27, p. 293.

20. Andersson, E.; Barnett, S. M.; Aspect, A. Joint measurements of spin, operational locality and uncertainty. Phys. Rev. A 2005, 72, 042104.

21. Wolf, M. M.; Perez-Garcia, D.; Fernandez, C. Measurements incompatible in quantum theory cannot be measured jointly in any other no-signaling theory. Phys. Rev. Lett. 2009, 103, 230402.

22. Cushing, J.; McMullin, E. (eds). Philosophical Consequences of Quantum Theory: Reflections on Bell's Theorem; Notre Dame, 1989.

23. Van der Merwe, A.; Selleri, F.; Tarozzi, G. (eds). Bell's Theorem and the Foundations of Modern Physics; World Scientific, 1992.

24. Fine, A. Joint distributions, quantum correlations, and commuting observables. J. Math. Phys. 1982, 23, p. 1306.

25. Eibl, M.; et al. Experimental observation of four-photon entanglement from parametric down-conversion. Phys. Rev. Lett. 2003, 90, 200403.

26. Pan, J.; et al. Experimental demonstration of four-photon entanglement and high-fidelity teleportation. Phys. Rev. Lett. 2001, 86, p. 4435.

27. Pan, J.; et al. Multiphoton entanglement and interferometry. Rev. Mod. Phys. 2012, 84, p. 777.

28. Kim, Y. H.; Shih, Y. Experimental realization of Popper's experiment: violation of the uncertainty principle? Found. Phys. 1999, 29, p. 1849.

29. Shih, Y. The physics of 2 is not 1+1. The Western Ontario Series in Philosophy of Science 2009, 73, p. 157.

30. Bohr, N. Collected Works, Vol. 3: The correspondence Principle (1918-1923); North-Holland, 1976. 